\title{
Nutritional composition and total collagen content of three commercially important edible jellyfish
}

\begin{abstract}
The study aimed to evaluate nutraceutical potential of three commercially significant edible jellyfish species (Acromitus hardenbergi, Rhopilema hispidum and Rhopilema esculentum). The bell and oral arms of these jellyfishes were analyzed for their proximate composition, calorific value, collagen content, amino acid profile, chemical score and elemental constituent. In general, all jellyfish possessed low calorific values (1.0ї $4.9 \mathrm{kcal} / \mathrm{g} \mathrm{D.W}$.) and negligible fat contents (0.4ї $1.8 \mathrm{~g} / 100 \mathrm{~g} \mathrm{D.W}$.), while protein (20.0ї 53.9 g/100 g D.W.) and minerals (15.9ï $57.2 \mathrm{~g} / 100 \mathrm{~g} \mathrm{D.W}$.) were found to be the richest components. Total collagen content of edible jellyfish varied from 122.64 to $693.92 \mathrm{mg} / \mathrm{g}$ D.W., accounting for approximately half its total protein content. The dominant amino acids in both bell and oral arms of all jellyfish studied includes glycine, glutamate, threonine, proline, aspartate and arginine, while the major elements were sodium, potassium, chlorine, magnesium, sulfur, zinc and silicon. Among the jellyfish, A. hardenbergi exhibited significantly higher total amino acids, chemical scores and collagen content $(\mathrm{p}<0.05)$ compared to R. hispidum and R. esculentum. Having good protein quality and low calories, edible jellyfish is an appealing source of nutritive ingredients for the development of oral formulations, nutricosmetics and functional food.
\end{abstract}

Keyword: Edible jellyfish; Acromitus hardenbergi; Rhopilema hispidum; Rhopilema esculentum; Nutritional composition; Amino acid composition; Collagen content; Elemental composition 